\title{
Antinociceptive effects of fucoidan in rat models of vincristine-induced neuropathic pain
}

\author{
CHUANYIN HU ${ }^{1}$, YUN-TAO ZHAO ${ }^{2}$, GUOPING ZHANG ${ }^{1}$ and MING-FENG XU ${ }^{3}$ \\ ${ }^{1}$ Department of Biology, Guangdong Medical University, Zhanjiang, Guangdong 524023; \\ ${ }^{2}$ Modern Biochemistry Center, Guangdong Ocean University, Zhanjiang, Guangdong 524088; \\ ${ }^{3}$ Department of Physiology, Guangdong Medical University, Zhanjiang, Guangdong 524023, P.R. China
}

Received November 30, 2015; Accepted November 22, 2016

DOI: $10.3892 / \mathrm{mmr} .2016 .6071$

\begin{abstract}
Chemotherapeutic drugs commonly induce peripheral neuropathic pain, which limit their clinic use. In the present study, the effect of fucoidan on the development of vincristine-induced neuropathic pain was evaluated and the underlying mechanism was examined. A neuropathy model was established in Sprague-Dawley rats by intraperitoneal injection of vincristine sulfate $50 \mu \mathrm{g} / \mathrm{kg}$ once a day for 10 consecutive days. Fucoidan (50, 100 or $200 \mathrm{mg} / \mathrm{kg}$.) and pregabalin $(10 \mathrm{mg} / \mathrm{kg})$ were injected for 14 consecutive days. Behavioral assessments were then performed and the expression of GABAB receptor was determined. The results showed that a single treatment with fucoidan did not prevent the induction of vincristine-induced mechanical or cold allodynia. However, repeated fucoidan administration attenuated vincristine-induced mechanical and cold allodynia in a dose-dependent manner. Additionally, the analgesic effects of fucoidan contributed to an upregulation in the expression of GABAB receptor in the spinal cord. Furthermore, all the effects of fucoidan against vincristine-induced neuropathy were reversed by saclofen, a selective GABAB receptor antagonist. These results suggested that the antinociceptive effects of fucoidan may be through activation of GABAB receptor, and fucoidan may be a promising drug for the treatment of chemotherapeutic drug-induced neuropathic pain.
\end{abstract}

\section{Introduction}

Chemotherapeutic agents frequently produce peripheral neuropathic pain as their primary side effect. Vincristine, which is one of the most common chemotherapeutic drugs, is used to treat a variety of types of cancer. However, the development of neuropathy induced by vincristine chemotherapy not only

Correspondence to: Dr Ming-Feng Xu, Department of Physiology, Guangdong Medical University, 2 East Wenming Road, Zhanjiang, Guangdong 524023, P.R. China

E-mail: xumf6548@126.com

Key words: antinociceptive, chemotherapy, allodynia, GABAB receptor, fucoidan leads to dose reduction or discontinuation of treatment, but also decreases the quality of life in cancer survivors (1).

Conventional analgesics, including tricyclic antidepressants, anticonvulsants, opioids, nonsteroidal anti-inflammatory drugs, and $\alpha 2$-adrenoceptor agonists have been prescribed for the treatment of neuropathic pain $(2,3)$. However, these medications have also been found to exhibit a wide spectrum of adverse effects, which limit their full clinical exploitation. Various herbal medicines have been documented to have therapeutic potential for the management of neuropathic pain (4). Clinical studies have also reported the beneficial effects of herbal medicines in cases of neuropathic pain $(5,6)$. Therefore, the investigation of novel herbal medicines in the management of neuropathic pain is urgently required.

Fucoidan is a complex sulfated polysaccharide, derived from marine brown seaweed, which has been reported to have anticoagulant, antithrombotic, antiviral antitumor, antioxidant and anti-inflammatory effects $(7,8)$. It is readily available from several marine algae species and is considered as a functional food, which may exert systemic effects following oral administration. Our previous investigations demonstrated that fucoidan attenuates allodynia and hyperalgesia in spinal nerve ligation-induced neuropathic pain (9). However, it is unclear whether it would relieve chemotherapy-induced neuropathic pain. In the present study, it was observed that fucoidan exerted an analgesic effect in an animal model of vincristine-induced neuropathic pain. It was also found that the GABAB receptor is possibly involved in the antinociceptive effects of fucoidan on neuropathic pain.

\section{Materials and methods}

Animals. Male SPF Sprague-Dawley rats (200-250 g) were obtained from the Experimental Animal Center of Guangdong Medical University (Guangdong, China). All animals were maintained on a $12 \mathrm{~h}$ light/dark cycle at a room temperature of $22 \pm 1^{\circ} \mathrm{C}$ with food and water available ad libitum. All experimental protocols described in the present study were approved by the Animal Care and Use Committee at Guangdong Medical University and conformed to the NIH guidelines (10).

Induction of neuropathic pain by vincristine. Peripheral neuropathic pain was induced in the rats via the administration 
of $50 \mu \mathrm{g} / \mathrm{kg}$ vincristine sulfate intraperitoneally (i.p.) for 10 consecutive days, as described by Siau and Bennett (11).

Intrathecal implantation. Animals were anesthetized with $60 \mathrm{mg} / \mathrm{kg}$, intraperitoneal injection (i.p.) of sodium pentobarbital. A polyethylene (PE)-10 tube (BD Biosciences, San Jose, CA, USA) was implanted into the subarachnoid space of the lumbar enlargement for intrathecal drug administration. The catheter placement was verified by observing transient hindpaw paralysis induced by intrathecal injection of $2 \%$ lidocaine $(10 \mu \mathrm{l})$. Only those rats showing complete paralysis of both hind limbs and the tail following the administration of lidocaine were used for the subsequent experiments. At the end of each experiment, the position of the PE tubing in the intrathecal space at the lumbar enlargement was visually verified by exposing the lumbar spinal cord.

Drug administration. Vincristine sulfate (Hisun Pharmaceutical Co., Ltd., Zhejiang, China) was dissolved in normal saline $(0.9 \% \mathrm{NaCl})$. Fucoidan from Fucus vesiculosus (Sigma-Aldrich; Merck Millipore, Darmstadt, Germany) was dissolved in normal saline $(0.9 \% \mathrm{NaCl})$. Different doses of fucoidan (50, 100 and $200 \mathrm{mg} / \mathrm{kg}$ ) were injected i.p. The day prior to the first administration of vincristine was considered to be day 0 . The investigation was divided into three experiments. In the single treatment experiment, fucoidan $(50,100$ or $200 \mathrm{mg} / \mathrm{kg}$ ) or normal saline was administered to the vincristine-treated rats once on day $14(n=6$ rats per treatment group). Behavioral assessments were performed on day 14 immediately prior to administration of the drug, and at $2,12 \mathrm{~h}$, 1 day, 3 days and 7 days post-administration.

In the repetitive treatment experiment, the animals were divided into six groups for administration: Rats were administered with normal saline for 14 consecutive days as a control group; vincristine-treated rats were administered with fucoidan $(50,100$ or $200 \mathrm{mg} / \mathrm{kg}$ i.p.) or normal saline once daily for 14 consecutive days; pregabalin (Sigma-Aldrich; Merck Millipore) was dissolved in normal saline and $10 \mathrm{mg} / \mathrm{kg}$ was injected (i.p.) for 14 consecutive days in the vincristine-treated rats as a positive control $(n=6$ rats per treatment group). Behavioral assessments were performed on days $0,1,3,7,14$ and 21 .

To evaluate the involvement of GABAB receptor in the analgesic effect of fucoidan, a third experiment was performed. Based on the results obtained in the repetitive treatment experiment, a $200 \mathrm{mg} / \mathrm{kg} / \mathrm{day}$ dose of fucoidan was selected for further investigations. The vincristine-treated rats were administered with $200 \mathrm{mg} / \mathrm{kg}$ fucoidan or normal saline once daily for 14 consecutive days. Saclofen (Sigma-Aldrich; Merck Millipore) was diluted to a concentration of $1 \mu \mathrm{g} / \mu \mathrm{l}$ in saline for intrathecal injection. Between days 15 and $21,10 \mu \mathrm{l}$ of saclofen or saline were administered once daily for 7 consecutive days. A total of 24 rats were equally randomized into four groups: i) Vincristine-saline-saline treatment; ii) vincristine-fucoidan-saline treatment; iii) vincristine-saline-saclofen treatment; (4) vincristine-fucoidan-saclofen treatment. Behavioral assessments were performed on days 14 and 21.

Mechanical paw withdrawal assessment. Mechanical allodynia was assessed using von Frey filaments (Stoelting,
Kiel, WI, USA) by experimenters who were blinded to the group assignment. The ipsilateral hind paw was pressed with one of a series of von Frey filaments with gradually increasing rigidity $(2,4,6,8,10,15$ and $20 \mathrm{~g})$, each of which was applied to the plantar surface for 5-6 sec. A positive paw withdrawal response was recorded if the animal briskly lifted the hindpaw. The mechanical withdrawal threshold was determined using the up-down method (12).

Cold allodynia assessment. Cold allodynia was assessed by placing $100 \mu \mathrm{l}$ of acetone on the planter surface of the left hind paw of the rat. A cold chemical sensitive reaction with respect to either paw licking, shaking or rubbing the left hind paw was observed and recorded as the paw withdrawal threshold. The cut-off duration of $20 \mathrm{sec}$ was maintained.

Western blot analysis. Following the behavioral assessments on day 21 , the rats were anesthetized with chloral hydrate (300 mg/kg, i.p.), perfused intracardially with $250 \mathrm{ml}$ cold saline and then sacrificed by decapitation. The L4-6 spinal cord was promptly removed onto an ice-cold plate, frozen and stored at $-80^{\circ} \mathrm{C}$ until use. All collected tissue samples were homogenized in an SDS sample buffer containing a mixture of proteinase and phosphatase inhibitors (Sigma-Aldrich; Merck Millipore). Each individual rat spinal cord protein was processed separately. The protein concentrations were estimated using the bicinchoninic acid method. Protein samples $(30 \mu \mathrm{g})$ were separated by $10 \%$ SDS-PAGE gel and transferred onto a nitrocellulose membrane. The membranes were blocked in $10 \%$ skimmed milk for $1 \mathrm{~h}$ at room temperature, and then incubated with the following primary antibodies overnight at $4^{\circ} \mathrm{C}$ : Guinea pig polyclonal anti-GABAB2 (1:2,000; cat. no. AB2255; Chemicon, Temecula,CA, USA) and mouse monoclonal anti- $\beta$-actin (1:3,000; cat. no. A1978; Sigma-Aldrich; Merck Millipore). The blots were further incubated for $1 \mathrm{~h}$ at room temperature with HRP-conjugated secondary antibody (anti-guinea pig 1:3,000; cat. no. 61_4620; anti-mouse cat. no. 31430; 1:5,000; GE Healthcare Life Sciences, Chalfont, UK), and washed three times in TBS for $30 \mathrm{~min}$. The immune complexes were detected using the enhanced chemiluminescence detection method (GE Healthcare Life Sciences) and exposure to film. A square of the same size was drawn around each band to measure the density, and the background adjacent to that band was subtracted. Levels of the target protein were normalized against the levels of $\beta$-actin and expressed as relative fold changes.

Statistical analysis. Values are expressed as the mean \pm standard error of the mean. For behavioral data, comparisons were made using two-way repeated measures analysis of variance (ANOVA). For others, comparisons were performed using two-way ANOVA followed by Bonferroni tests. Data were analyzed using SPSS 13.0 (SPSS, Inc., Chicago, IL, USA). $\mathrm{P}<0.05$ was considered to indicate a statistically significant difference.

\section{Results}

Single treatment of fucoidan on vincristine-induced mechanical and cold allodynia. Vincristine administration resulted in 


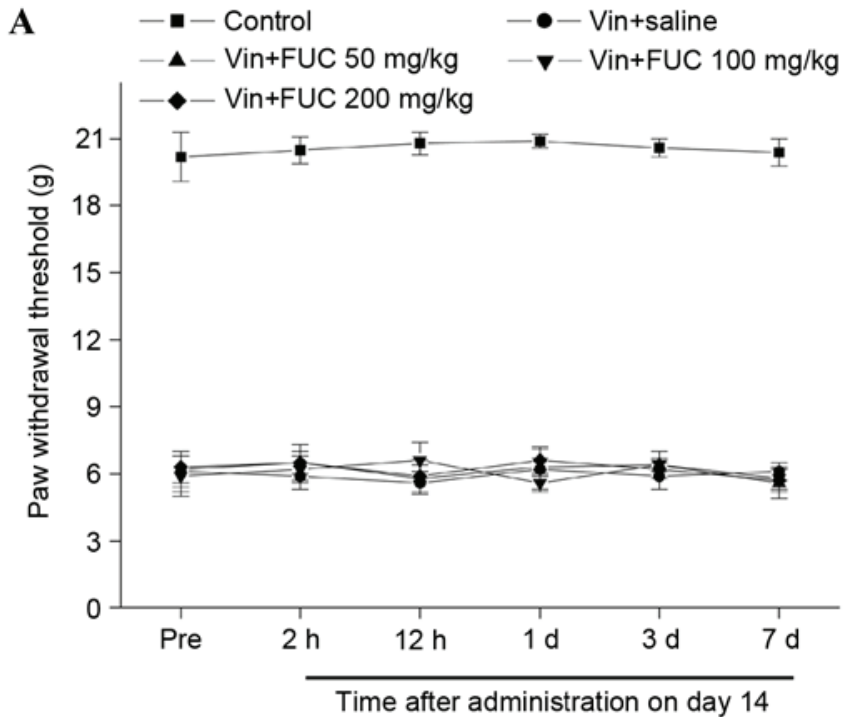

B

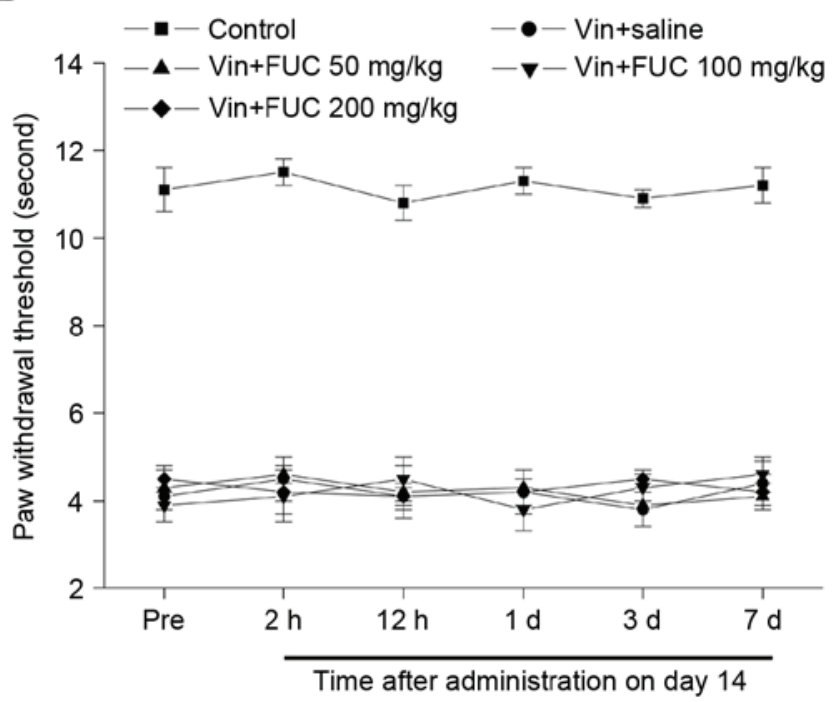

Figure 1. Single treatment with fucoidan has no effect on vincristine-induced mechanical and cold allodynia. Vincristine $(50 \mu \mathrm{g} / \mathrm{kg})$ was injected (i.p.) for 10 consecutive days. Fucoidan was injected once (i.p.) on day 14. The behavioral response was evaluated 2, 12 h, 1 day, 3 days and 7 days post-injection. Vincristine administration resulted in prominent (A) mechanical and (B) cold allodynia on day 14 . However, single treatment with fucoidan did not reverse vincristine-induced mechanical or cold allodynia ( $n=6$ in each group). Vin, vincristine; FUC, fucoidan.

the development of mechanical and cold allodynia, as reflected by the significant reductions in the mechanical and cold withdrawal thresholds, compared with the normal control group. To determine the effects of a single injection of fucoidan on vincristine-induced allodynia, fucoidan was injected i.p. on day 14 , when the neuropathic pain had been maintained. The behavioral responses 2, 12 h, 1 day, 3 days and 7 days following administration were then observed. No differences in mechanical or cold allodynia were detected between the rat treated with fucoidan and normal saline, at any doses or time points, following injection (Fig. 1A and B).

Repeated injection of fucoidan on vincristine-induced mechanical and cold allodynia. The effects of repeated fucoidan treatment on vincristine-induced neuropathic pain
A

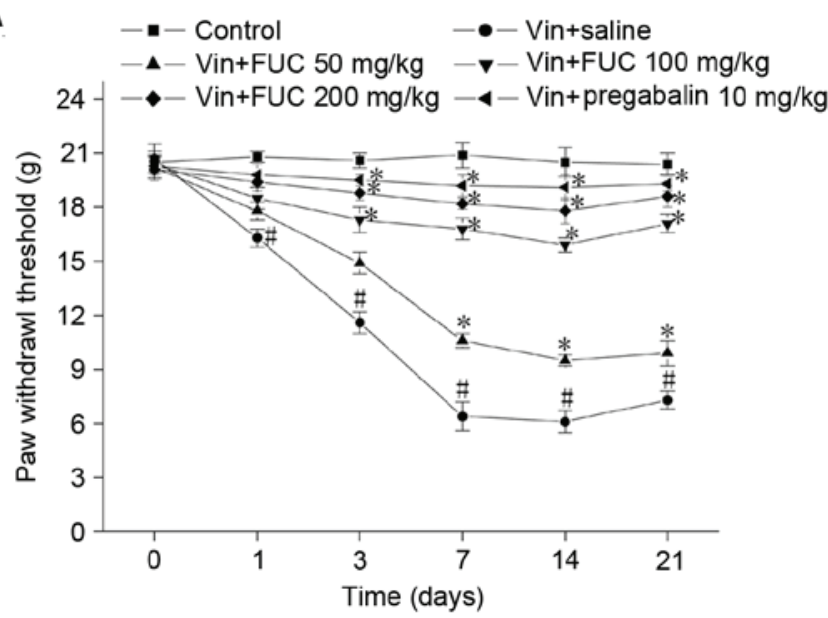

B

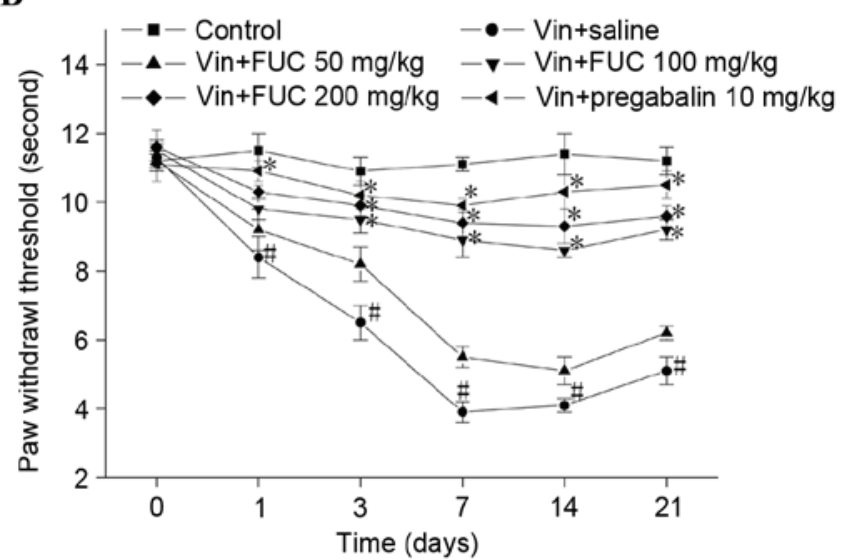

Figure 2. Effects of repeated treatment with fucoidan on vincristine-induced mechanical and cold allodynia. Vincristine $(50 \mu \mathrm{g} / \mathrm{kg}$ ) was injected (i.p.) for 10 consecutive days. Fucoidan $(50,100$ or $200 \mathrm{mg} / \mathrm{kg}$ ) and normal saline or pregabalin $(10 \mathrm{mg} / \mathrm{kg})$ were administered once daily for 14 consecutive days in vincristine-treated rats. Rats were administered with normal saline for 14 consecutive days as a control. Behavioral assessments were performed on days $0,1,3,7,14$ and 21 . Following treatment with fucoidan, (A) mechanical and (B) cold paw withdrawal threshold in the $\mathrm{Vin}+\mathrm{FUC}$ group were significantly higher, compared with those of the Vin+saline group at each relative time point. The effect of fucoidan administration on vincristine-induced neuropathic pain was dose-dependent ( $\mathrm{n}=6$ in each group). ${ }^{*} \mathrm{P}<0.05$, compared with the Vin+saline group; ${ }^{\#} \mathrm{P}<0.05$, compared with the control. Vin, vincristine; FUC, fucoidan.

were also examined. As shown in Fig. 2A and B, repeated administration of fucoidan attenuated vincristine-induced mechanical and cold allodynia in a dose-dependent manner. A lower dose of fucoidan $(50 \mathrm{mg} / \mathrm{kg})$ only marginally elevated the mechanical and cold paw withdrawal threshold. Higher doses of fucoidan (100 and $200 \mathrm{mg} / \mathrm{kg}$ ) markedly and significantly increased the mechanical and cold paw withdrawal threshold. Similar effects were observed with pregabalin administration. In addition, a higher dose of fucoidan ameliorated vincristine-induced neuropathic pain for at least 1 week following the final treatment.

Effects of repeated fucoidan administration on the expression of $G A B A B$ receptor in the spinal cord of vincristine-treated rats. $\mathrm{GABAB}$ receptor is densely expressed in the spinal cord dorsal horn. Activation of spinal GABAB receptor produces 
A

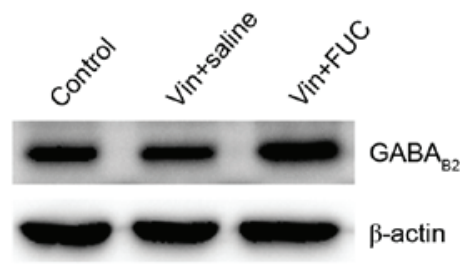

B

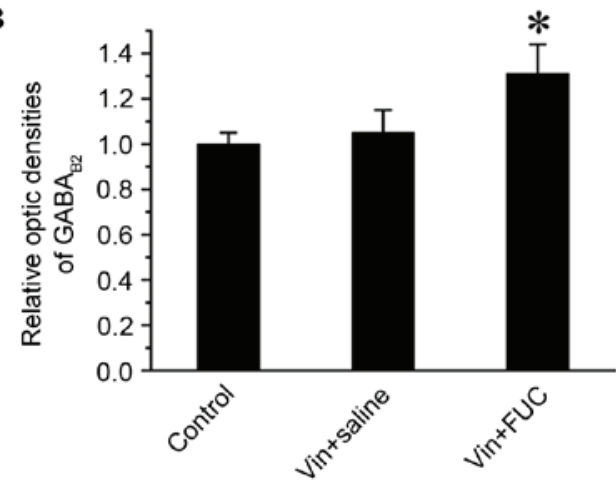

Figure 3. Chronic fucoidan treatment increases the expression of GABAB2 receptor in the spinal cord. The expression of GABAB2 receptor was revealed using western blot analysis. Vincristine did not alter the expression of GABAB2 receptor, compared with the control. Treatment with $200 \mathrm{mg} / \mathrm{kg}$ fucoidan significantly increased the expression of GABAB2 receptor ( $\mathrm{n}=4$ in each group). ${ }^{*} \mathrm{P}<0.05$ compared with the control. Results are expressed as fold changes relative to the control. Vin, vincristine; FUC, fucoidan.
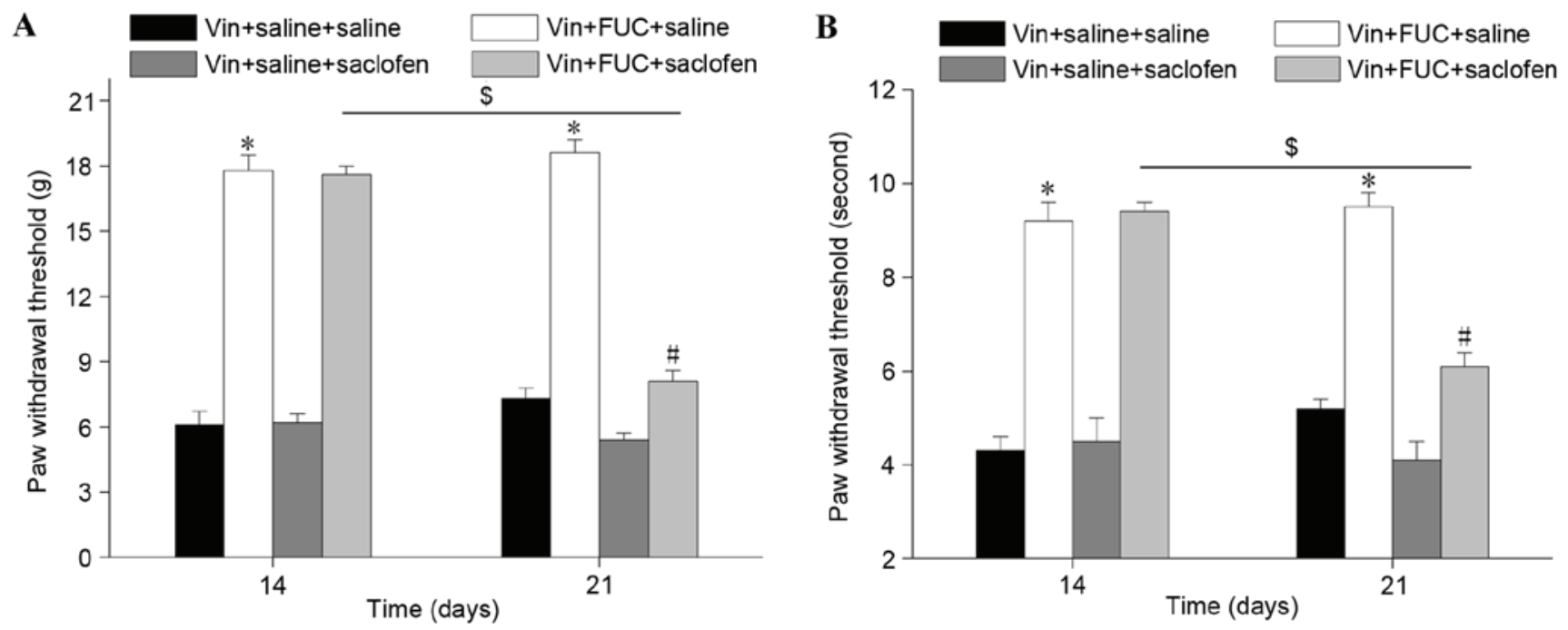

Figure 4. Analgesic effect of fucoidan is reversed by intrathecal administration of a GABAB antagonist. Vincristine (50 $\mu \mathrm{g} / \mathrm{kg})$ was injected (i.p.) for 10 consecutive days. Fucoidan $(200 \mathrm{mg} / \mathrm{kg}$ ) or normal saline were administered for 14 consecutive days in the vincristine-treated rats. Rats were injected intrathecally with saclofen or saline once per day for 7 days (day 15-21) following fucoidan treatment. (A) Mechanical and (B) cold paw withdrawal thresholds were assessed on days 14 and 21. The results confirmed the analgesic action of fucoidan and showed that saclofen completely reversed the analgesic action on (A) mechanical and (B) cold allodynia ( $\mathrm{n}=6$ in each group). " $\mathrm{P}<0.05$, Vin-FUC-saline, vs. Vin-saline-saline; ${ }^{*} \mathrm{P}<0.05$, Vin-FUC-saclofen, vs. Vin-FUC-saline; ${ }^{\$} \mathrm{P}<0.05$, Vin-FUC-saclofen. Vin, vincristine; FUC, fucoidan.
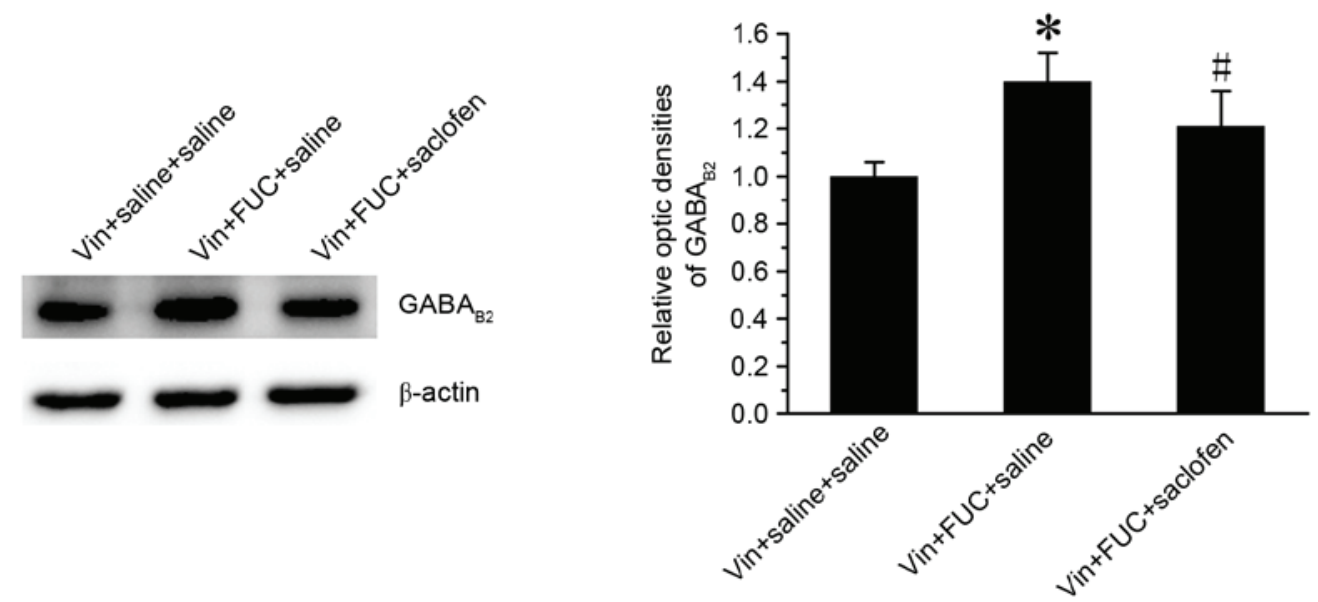

Figure 5. Effects of saclofen on the effect of fucoidan on the expression of GABAB2 receptor in the spinal cord of vincristine-treated rats. Western blot analysis showed that saclofen reversed the upregulated GABAB2 receptor induced by fucoidan ( $\mathrm{n}=4$ in each group). $\mathrm{P}<0.05$, Vin-FUC-saline, vs. Vin-saline-saline; ${ }^{\#} \mathrm{P}<0.05$, Vin-FUC-saclofen, vs, Vin-FUC-saline. Results are expressed as fold changes relative to the Vin-saline-saline group. Vin, vincristine; FUC, fucoidan. 
antinociception in acute and chronic pain $(13,14)$. A study by Thibault et al (15) reported that the GABAB receptor is functionally required for the alleviating effect of oxycodone in vincristine-induced neuropathic pain. The analgesic effect of fucoidan on vincristine-induced neuropathic pain may be associated with its upregulation of the expression of GABAB receptor. To confirm this hypothesis, the present study examined the expression levels of GABAB2 receptor using western blot analysis. The data showed that there was an upregulation in the expression of GABAB2 receptor in the vincristine-fucoidan treated animals, compared with the expression in the animals in the vincristine-saline treated animals or the saline-treated animals on 21 (Fig. 3A and B).

Effect of the GABAB receptor on the analgesic effect of repeated fucoidan injections determined by intrathecal administration of saclofen. The present study directly examined the role of GABAB receptor in the analgesic effect of fucoidan by assessing the effect of the intrathecal administration of saclofen, a selective GABAB receptor antagonist. As shown in Fig. 4A and B, the alleviating effect of repeated fucoidan administration on vincristine-induced mechanical and cold allodynia was completely reversed by the antagonist. Furthermore, saclofen eliminated the effect of fucoidan on the upregulated expression of GABAB2 receptor in the spinal cord (Fig. 5). Of note, the antinoticeptive effect of fucoidan $(200 \mathrm{mg} / \mathrm{kg})$ was maintained 7 days following the final administration. These results revealed that fucoidan had a prolonged effect via the upregulation of GABAB receptor, which can be observed even when fucoidan treatment is terminated.

\section{Discussion}

GABAB receptors are located both pre- and post-synaptically throughout the central nervous system. GABAB receptor stimulation affects neurotransmission by directly reducing neurotransmitter release or hyperpolarizing postsynaptic neurons (16). Increasing GABAergic tonus decreases neuronal excitation and has an antinociceptive property. For example, Baclofen, the prototypic, orthosteric GABAB receptor agonist, produces antinociception in neuropathic pain models whether administered intrathecally or intraperitoneally and has been used clinically as an adjuvant therapy for managing certain types of pain $(13,17,18)$. In the present study, it was observed that repeated administration of fucoidan significantly attenuated vincristine-induced mechanical and cold allodynia. Of note, the antinociceptive effect of fucoidan was not detected following a single injection of fucoidan. In addition, fucoidan treatment induced an increase in GABAergic tone in the spinal cord, which was mediated by the GABAB2 receptor. As the effect of this Chinese herb extract usually has a long duration to onset, repetitive treatments of fucoidan are required to exert the effect of antinociception. The present study hypothesized that the upregulated expression of GABAB2 receptor following fucoidan administration led to a decrease in excitatory transmission and counteracted the increased neuronal excitability induced by vincristine treatment. This hypothesis was confirmed by the use of saclofen, a selective GABAB receptor antagonist, which reversed the effects of fucoidan $(200 \mathrm{mg} / \mathrm{kg})$ against vincristine-induced neuropathy.
Therefore, the alleviating effect of fucoidan in neuropathic pain appeared to involve the spinal GABAergic tonus through the GABAB receptor. However, how fucoidan increases the expression of $\mathrm{GABAB}$ receptor remains to be elucidated. Glutamate is a major excitatory transmitter in the spinal cord and N-methyl-D-aspartate receptor (NMDAR) is known to be involved in painful neuropathy. Activation of the GABAB receptor can downregulate NMDAR in the spinal cord in diabetic neuropathy (19), whereas NMDAR is upregulated in chemotherapeutic drug oxaliplatin-induced neuropathic pain (20). Thus it was hypothesized that the fucoidan-induced increase in GABAB receptor occurred through the downregulation of NMDAR. However, further investigations are required to confirm this hypothesis.

Previous studies have shown that a decline in the expression of GABAB receptor in the spinal cord is associated with certain types of neuropathic pain. For example, nerve injury-induced neuropathic pain has been found to cause upregulation, downregulation, and no effect on the expression of GABAB receptor (21-23). GABAB receptor is downregulated in the spinal dorsal horn in the rat models of streptozotocin-induced diabetic neuropathic pain (24). However, vincristine treatment was found not to effect the expression of GABAB2 receptor in the spinal dorsal horn of rats with chemotherapy-induced neuropathic pain (14). The present study also observed no alteration in the expression of GABAB2 in vincristine-treated rats. These data suggested that an association between the development of neuropathic pain and a decline in spinal cord GABAergic activity was not always observed, possibly due to differences in the animal models or in the route or pattern of drug administration. There is substantial evidence indicating that the systemic administration of orthosteric GABAB receptor agonist generally produces antinociception in animal models of acute and persistent pain $(13,25,26)$. Therefore, agents that activate GABAB receptor may provide antinociceptive effects. The results of the present study suggested that the repeated administration of fucoidan significantly ameliorated vincristine-induced mechanical and cold allodynia, possibly through activation of the GABAB receptor. Therefore, fucoidan may be a promising drug for the treatment of chemotherapeutic drug-induced neuropathic pain.

\section{Acknowledgements}

The present study was supported by the Science and Technology Projects of Guangdong Province (grant no. 2014A020212507).

\section{References}

1. Dougherty PM, Cata JP, Burton AW, Vu K and Weng HR: Dysfunction in multiple primary afferent fiber subtypes revealed by quantitative sensory testing in patients with chronic vincristine-induced pain. J Pain Symptom Manage 33: 166-179, 2007.

2. Dworkin RH, O'Connor AB, Audette J, Baron R, Gourlay GK, Haanpää ML, Kent JL, Krane EJ, Lebel AA, Levy RM, et al: Recommendations for the pharmacological management of neuropathic pain: An overview and literature update. Mayo Clin Proc 85 (Suppl 3): S3-S14, 2010.

3. Lee $J$ and Nandi P: Improving the management of neuropathic pain. Practitioner 254: 27-30, 3, 2010.

4. Kim YS, Park HJ, Kim TK, Moon DE and Lee HJ: The effects of Ginkgo biloba extract EGb 761 on mechanical and cold allodynia in a rat model of neuropathic pain. Anesth Analg 108: 1958-1963, 2009. 
5. Nurmikko TJ, Serpell MG, Hoggart B, Toomey PJ, Morlion BJ and Haines D: Sativex successfully treats neuropathic pain characterised by allodynia: A randomised, double-blind, placebo-controlled clinical trial. Pain 133: 210-220, 2007.

6. Ellis RJ, Toperoff W, Vaida F, van den Brande G, Gonzales J, Gouaux B, Bentley H and Atkinson JH: Smoked medicinal cannabis for neuropathic pain in HIV: A randomized, crossover clinical trial. Neuropsychopharmacology 34: 672-680, 2009.

7. Wang J, Zhang Q, Zhang Z, Song H and Li P: Potential antioxidant and anticoagulant capacity of low molecular weight fucoidan fractions extracted from Laminaria japonica. Int J Biol Macromol 46: 6-12, 2010.

8. Aisa Y, Miyakawa Y, Nakazato T, Shibata H, Saito K, Ikeda Y and Kizaki M: Fucoidan induces apoptosis of human HS-sultan cells accompanied by activation of caspase- 3 and down-regulation of ERK pathways. Am J Hematol 78: 7-14, 2005.

9. Hu CY, Zhang GP and Zhao YT: Fucoidan attenuates the existing allodynia and hyperalgesia in a rat model of neuropathic pain. Neurosci Lett 571: 66-71, 2014

10. National Institutes of Health (NIH): Office of Laboratory Anima Welfare (OLAW). http://olaw.nih.gov. Accessed May 20, 2016.

11. Siau C and Bennett GJ: Dysregulation of cellular calcium homeostasis in chemotherapy-evoked painful peripheral neuropathy. Anesth Analg 102: 1485-1490, 2006.

12. Chaplan SR, Bach FW, Pogrel JW, Chung JM and Yaksh TL: Quantitative assessment of tactile allodynia in the rat paw. J Neurosci Methods 53: 55-63, 1994.

13. Smith GD, Harrison SM, Birch PJ, Elliott PJ, Malcangio M and Bowery NG: Increased sensitivity to the antinociceptive activity of (+/-)-baclofen in an animal model of chronic neuropathic, but not chronic inflammatory hyperalgesia. Neuropharmacology 33: 1103-1108, 1994.

14. Dirig DM and Yaksh TL: Intrathecal baclofen and muscimol, but not midazolam, are antinociceptive using the rat-formalin model. J Pharmacol Exp Ther 275: 219-227, 1995.

15. Thibault K, Calvino B, Rivals I, Marchand F, Dubacq S, McMahon SB and Pezet S: Molecular mechanisms underlying the enhanced analgesic effect of oxycodone compared to morphine in chemotherapy-induced neuropathic pain. PLoS One 9: e91297, 2014.
16. Brenowitz S, David J and Trussell L: Enhancement of synaptic efficacy by presynaptic GABA(B) receptors. Neuron 20: 135-141, 1998.

17. Hwang JH, Hwang KS, Kim JU, Choi IC, Park PH and Han SM: The interaction between intrathecal neostigmine and GABA receptor agonists in rats with nerve ligation injury. Anesth Analg 93: 1297-1303, 2001.

18. Slonimski M, Abram SE and Zuniga RE: Intrathecal baclofen in pain management. Reg Anesth Pain Med 29: 269-276, 2004.

19. Bai HP, Liu P, Wu YM, Guo WY, Guo YX and Wang XL: Activation of spinal GABAB receptors normalizes $\mathrm{N}$-methyl-D-aspartate receptor in diabetic neuropathy. J Neurol Sci 341: 68-72, 2014

20. Liu XY, Zhang GF, Dong L, Wang X, Sun H, Shen J, Li W and Xu J: Repeated administration of mirtazapine attenuates oxaliplatin-induced mechanical allodynia and spinal NR2B up-regulation in rats. Neurochem Res 38: 1973-1979, 2013.

21. McCarson KE, Ralya A, Reisman SA and Enna SJ: Amitriptyline prevents thermal hyperalgesia and modifications in rat spinal cord GABA(B) receptor expression and function in an animal model of neuropathic pain. Biochem Pharmacol 71: 196-202, 2005.

22. Castro-Lopes JM, Malcangio M, Pan BH and Bowery NG: Complex changes of GABAA and GABAB receptor binding in the spinal cord dorsal horn following peripheral inflammation or neurectomy. Brain Res 679: 289-297, 1995.

23. Engle MP, Gassman M, Sykes KT, Bettler B and Hammond DL: Spinal nerve ligation does not alter the expression or function of $\mathrm{GABA}(\mathrm{B})$ receptors in spinal cord and dorsal root ganglia of the rat. Neuroscience 138: 1277-1287, 2006.

24. Wang XL, Zhang Q, Zhang YZ, Liu YT, Dong R, Wang QJ and Guo YX: Downregulation of GABAB receptors in the spinal cord dorsal horn in diabetic neuropathy. Neurosci Lett 490: $112-115,2011$.

25. McCarson KE and Enna SJ: GABA pharmacology: The search for analgesics. Neurochem Res 39: 1948-1963, 2014.

26. Thomas DA, Navarrete IM, Graham BA, McGowan MK and Hammond DL: Antinociception produced by systemic $\mathrm{R}(+)$-baclofen hydrochloride is attenuated by CGP 35348 administered to the spinal cord or ventromedial medulla of rats. Brain Res 718: 129-137, 1996. 